\title{
Interview with a Thought Leader on Systems Medicine-Charles Auffray, PhD
}

\author{
President, European Institute for Systems Biology \& Medicine (EISBM), Lyon, France; \\ Chair of the Executive Board, European Association of Systems Medicine, Aachen, Germany.
}

Charles Auffray is CNRS Research Director at the Joliot Curie Transdisciplinary Laboratory and Ecole Normale Supérieure de Lyon, Founding Director of the European Institute for Systems Biology and Medicine (EISBM) and Chair of the European Association of Systems Medicine (EASyM).

Why Does Systems Medicine Matter? Systems medicine represents the engine for progress in biomedicine and healthcare in the 21 st century, and thus matters as much as chemistry and molecular biology did in the last two centuries of the past millennium, when there was a growing emphasis on the study of biological systems at the molecular level, which led to highly significant advances on our understanding of their structure and common functioning principles. In the process, it became increasingly clear that microorganisms, plants, animals, and humans are much more complex systems than those studied by classical chemistry and physics. To gain a deep understanding of the mechanisms underlying their behavior in physiology and disease, the analytical reductionist approach focusing on the identification of a limited number of elements was reaching its limits. It needed to be complemented with more global and integrative approaches based on systemic principles focusing on complex networks of interactions. Thus, over the first two decades of this century and millenium, systems biology approaches have been successfully developed using the mathematical and computational conceptual frameworks and tools developed in other scientific fields. Taking advantage of major technological and engineering advances, they now make it possible to combine high-dimensional functional genomics data with assessments of biological and environmental conditions through iterative statistical analyses, computational modeling, and experimental validation. Their application to human pathology, also integrating clinical and lifestyle assessments to decipher the mechanisms underlying the development of diseases, predicts their onset and provides a basis for their treatment, led to the emerging field of systems medicine, which is thus empowered to transform biomedical research, clinical practice, and drug development, triggering in the process a transition from a reactive to a proactive practice of medicine. ${ }^{1-3}$

\section{What Is the Main Gap?}

Now that the conceptual and methodological frameworks of systems biology are set and available for deployment, their successful implementation in systems medicine to address unmet medical needs is facing two major hurdles that will take time to overcome as they require very important and essential changes to occur. First, the training curricula of scientists, engineers, and medical doctors, which developed in isolation in each field and discipline, need to be much better integrated so that all are sufficiently acquainted with the others 
to work together effectively using a common language and framework for mutual understanding. Second, the ethical, legal, socioeconomic, and regulatory environment has developed based on the assumptions of the importance of single determining elements and population averaged values of biological parameters to explain normal or dysfunctional health, which now need to be complemented or replaced by the complex networks and trajectories that are meaningful for single individuals. In both cases, a profound cultural change is needed.

\section{How Can It Be Filled?}

The effective development of participatory, personalized, predictive, and preventive (P4) systems medicine requires harmonization of experimental and computational methods for data, information, and knowledge collection, storage, analysis, and sharing on a big data scale using high-performance cloud computing infrastructures ensuring data security and compliance with personal data and privacy protection regulations. The active participation of all stakeholders in these long-term transformations, including researchers and clinicians in academy and industry, regulatory and funding bodies, individuals, and patient organizations, is thus essential.

\section{What Will Be the Impact?}

The impact of systems medicine will be enormous for the entire healthcare system and its associated industries. Pioneering individuals who have managed to anticipate the occurrence of disease and take preventive measures through a regular assessment of their exposome monitored through connected mobile devices (environmental and occupational exposures, nutrition, sleep, exercise, and stress), clinicome (biological and clinical features), and integrome (metabolomics, proteomic, transcriptomic, epigenomic, genomic, and genetic features) provide the basis for effective implementation of systems P4 medicine. ${ }^{4,5}$ Recent advances have made it possible to scale up the monitoring of well-being, health, and disease through the collection of billions of data points for increasing number of individuals who are healthy, at risk of developing disease, or in the course of disease development, with the active participation of individuals through social networks. ${ }^{6,7}$ The development of an open standard protocol enabling full compliance with personal data and privacy protection regulations will catalyze the transformation of healthcare delivery and the transition toward emphasis on management of well-being. ${ }^{8}$ With the full deployment of systems medicine, through their monitor- ing over a long period of time, individuals will be provided with actionable recommendations to maintain their state of health and well-being, detect early events indicative of a risk or a transition to disease, enabling their management and reversal. The expectation is that expanding the monitoring from one to millions then billions of individuals over the next 25 years will trigger in one generation a reversal of the escalating costs of healthcare management, drug, and diagnostic development, providing the basis for a more cost-efficient and sustainable integrated healthcare system.

\section{Is There Anything Else Relevant to Systems Medicine That You Find Important to State?}

As in many other scientific and technological fields, the European Union has demonstrated its potential to play a leading role in the development of systems medicine. ${ }^{8}$ The European Commission has invested very significant resources during the past decades in the development of systems biology and systems medicine, that is, through the Coordinating Action for the implementation of Systems Medicine in Europe (CASyM), which led to the formation of the European Association of Systems Medicine (EASyM; http://easym.eu), and a call for action to make sense of big data in health research. ${ }^{9}$ The journal Systems Medicine will hopefully serve as a vehicle to echo the progress made in demonstrating the effectiveness of systems medicine principles, first in successful proof-of-concept pilot projects, then with deployment through renovated healthcare systems worldwide.

\section{References}

1. Auffray C, Chen Z, Hood L. Systems medicine: the future of medical genomics and heathcare. Genome Med. 2009;1:2.

2. Hood L, Balling R, Auffray C. Revolutionizing medicine in the 21st century through systems approaches. Biotechnol J. 2012;7:992-1001.

3. Wolkenhauer $\mathrm{O}$, Auffray $\mathrm{C}$, Jaster $\mathrm{R}$, et al. The road from systems biology to systems medicine. Pediatr Res. 2013;73:502-507.

4. Smarr L. Quantifying your body: a how-to guide from a systems biology perspective. Biotechnol J. 2012;7:980-991.

5. Chen R, Mias Gl, Li-Pook-Than J, et al. Personal omics profiling reveals dynamic molecular and medical phenotypes. Cell. 2012;148:1293-1307.

6. Hood L, Auffray C. Participatory medicine: a driving force for revolutionizing healthcare. Genome Med. 2013;5:110.

7. Price ND, Magis AT, Earls JC, et al. A wellness study of 108 individuals using personal, dense, dynamic data clouds. Nat Biotechnol. 2017;35:747-756.

8. Auffray C, Sagner M, Abdelhak S, et al. Viva Europa, a land of excellence in research and innovation for health and wellbeing. Prog Prev Med. 2017;2: e006.

9. Auffray C, Balling R, Barroso I, et al. Making sense of big data in health research: toward an EU action plan. Genome Med. 2016;8:71.

Cite this article as: Auffray $C(2018)$ Interview with a thought leader on systems medicine-Charles Auffray, PhD, Systems Medicine 1:1, 11-12, DOI: 10.1089/sysm.2018.29002.cau. 